\title{
The Musculoskeletal Disorders in Diabetic Patients and the Evaluation of their Relationship with Metabolic Parameters and Microvascular Complications
}

\author{
Pelin Edis ${ }^{1}$, Nilufer Ozdemir ${ }^{1}$, and Zeliha Hekimsoy ${ }^{1}$ \\ ${ }^{1}$ Manisa Celal Bayar University
}

August 19, 2021

\begin{abstract}
Background: Diabetes mellitus has been associated with many different musculoskeletal system problems. Objective: This study aims to show the prevalence of musculoskeletal disorders in patients with diabetes and to reveal their relationship to the metabolic parameters and microvascular complications. Methods: Seven hundred two diabetic patients who consecutively applied to our clinic between March 2017 and February 2018 were included in this prospective cross-sectional study. The relationship of the musculoskeletal disorders including carpal tunnel syndrome, Dupuytren's contracture, adhesive capsulitis, flexor tenosynovitis, limited joint mobility syndrome, Charcot arthropathy, diabetic foot and gout to the patients' age, gender, type of diabetes, duration of diabetes, metabolic parameters, and microvascular complications were evaluated. Results: Musculoskeletal system disorders were detected in $45.9 \%(\mathrm{n}=322)$ of the 702 diabetic patients evaluated in our study. The most common disorders were carpal tunnel syndrome 17.7\% ( $\mathrm{n}=124)$, Dupuytren's contracture 14.4\% ( $\mathrm{n}=101)$, and adhesive capsulitis $13.8 \%$ ( $\mathrm{n}=97$ ). A statistically significant relationship of the HbA1c level with Dupuytren's contracture, carpal tunnel syndrome, and adhesive capsulitis was detected $(\mathrm{p}<0.05)$. Conclusions: Patients' age, duration of diabetes, HbA1c level, and presence of microvascular complications are associated with musculoskeletal disorders in this population.
\end{abstract}

The Musculoskeletal Disorders in Diabetic Patients and the Evaluation of their Relationship with Metabolic Parameters and Microvascular Complications

\section{Abstract}

Background : Diabetes mellitus has been associated with many different musculoskeletal system problems. Objective : This study aims to show the prevalence of musculoskeletal disorders in patients with diabetes and to reveal their relationship to the metabolic parameters and microvascular complications.

Methods: Seven hundred two diabetic patients who consecutively applied to our clinic between March 2017 and February 2018 were included in this prospective cross-sectional study. The relationship of the musculoskeletal disorders including carpal tunnel syndrome, Dupuytren's contracture, adhesive capsulitis, flexor tenosynovitis, limited joint mobility syndrome, Charcot arthropathy, diabetic foot and gout to the patients' age, gender, type of diabetes, duration of diabetes, metabolic parameters, and microvascular complications were evaluated.

Results : Musculoskeletal system disorders were detected in $45.9 \%(\mathrm{n}=322)$ of the 702 diabetic patients evaluated in our study. The most common disorders were carpal tunnel syndrome $17.7 \%(\mathrm{n}=124)$, Dupuytren's contracture 14.4\% $(\mathrm{n}=101)$, and adhesive capsulitis $13.8 \%(\mathrm{n}=97)$. A statistically significant relationship of the HbA1c level with Dupuytren's contracture, carpal tunnel syndrome, and adhesive capsulitis was detected $(p<0.05)$. 
Conclusions : Patients' age, duration of diabetes, HbA1c level, and presence of microvascular complications are associated with musculoskeletal disorders in this population.

Keywords: Diabetes mellitus; Musculoskeletal Disorders; Carpal tunnel syndrome; Dupuytren's contracture; Adhesive capsulitis

\section{What's already known about this topic?}

- Diabetes mellitus has been associated with many different musculoskeletal system problems.

- Diabetes mellitus (DM) is a chronic and progressive metabolic disorder characterized by hyperglycemia due to defective insulin secretion and defective insulin action or both.

- Diabetes causes progressive complications such as retinopathy, nephropathy, and neuropathy in the long term.

- Musculoskeletal disorders are generally not diabetes-specific and can also be seen in the non-diabetic population.

\section{What does this article add?}

- Several musculoskeletal disorders have been associated with diabetes.

- Musculoskeletal system disorders were detected in 322 of the 702 individuals with diabetes evaluated in our study.

- The frequency of musculoskeletal disorders increased with the presence of microvascular complications.

- Our study also draws attention to various risk factors that are effective in musculoskeletal system involvement in diabetes.

\section{Introduction}

Diabetes mellitus (DM) is a chronic and progressive metabolic disorder characterized by hyperglycemia due to defective insulin secretion and defective insulin action or both. ${ }^{1}$ Diabetes causes progressive complications such as retinopathy, nephropathy, and neuropathy in the long term. Musculoskeletal disorders are generally not diabetes-specific and can also be seen in the non-diabetic population. However, its incidence has increased significantly in patients with diabetes. ${ }^{2}$

Musculoskeletal disorders in patients with diabetes include adhesive capsulitis, limited joint mobility syndrome (LJMS), Dupuytren's contracture (DC), flexor tenosynovitis (FT), carpal tunnel syndrome (CTS), diffuse idiopathic skeletal hyperostosis (DISH), Charcot arthropathy, diabetic muscle infarction, diabetic foot, gout and osteoporosis. ${ }^{3}$ The pathophysiology of musculoskeletal disorders has not been fully explained, and different mechanisms triggered by hyperglycemia are thought to be effective. Accompanying vasculopathy and neuropathy also cause changes in the connective tissue. ${ }^{4}$ Although the complications in the musculoskeletal system are prevalent, they are often overlooked. However, the presence of these complications affects the patients' quality of life, leading to pain and limitation of movement.

In this study, we aimed to determine the prevalence of diabetes-related musculoskeletal disorders in our patients and the relationship of the musculoskeletal disorders including carpal tunnel syndrome, Dupuytren's contracture, adhesive capsulitis, flexor tenosynovitis, limited joint mobility syndrome, Charcot arthropathy, diabetic foot, and gout to the patients' age, gender, type of diabetes, duration of diabetes, HbA1c level and microvascular complications.

\section{Material and Methods}

In this prospective, cross-sectional study, 702 patients with diabetes who applied to our endocrinology clinic and outpatient clinic between March 2017 and February 2018 were consecutively included in the study, and the musculoskeletal disorders were assessed by the same physician. The patients with chronic arthritis due to rheumatological diseases such as osteoarthritis and rheumatoid arthritis, and those with collagen tissue disease, patients under 18 years of age, and those diagnosed with gestational diabetes were not included in the study. 
Informed consent was obtained from each of the participants. The study was approved by the Ethics Committee of Celal Bayar University, Faculty of Medicine (\#20.478.486-54). A detailed history of the patients was taken, and a physical examination was performed. Subsequently, the measurements of height, weight, waist circumference, and blood pressure were made. Afterward, ophthalmic examination findings, electromyography (EMG) results, and laboratory data were recorded from the outpatient files.

The symptoms related to the musculoskeletal system were investigated in these patients. The presence of LJMS was checked by the prayer sign and tabletop sign. For the diagnosis of Dupuytren's contracture, palmar or digital nodules were examined for findings such as pretendinous cord and flexion contracture. In order to determine flexor tenosynovitis, the patients were asked whether their fingers lock in flexion or extension. Passive and active shoulder movements were evaluated. A foot examination was performed for the presence of Hallux valgus, claw toes, pes cavus, Charcot joint, and diabetic foot. The relationship of musculoskeletal disorders with metabolic parameters and complications of diabetes was also evaluated in the present study.

\section{Statistical Analysis}

The Statistical Packages for the Social Sciences version 15.0 (SPSS Inc., Chicago, IL, USA) for Windows (Microsoft) was used for the data analysis. The data obtained in the study were given as mean \pm standard deviation (SD). Sociodemographic and diagnostic variables were shown in both numbers and percentages. The Student t-test was used for comparing two independent groups with normal distribution, One-way ANOVA (Analysis of Variance) for comparing more than two independent groups, and the Mann Whitney U test was used to compare two independent groups for variables without normal distribution. The relationship between categorical variables was tested by chi-square analysis. A $p$-value of less than 0.05 was accepted as statistically significant.

\section{Results}

The average age of the 702 diabetic patients evaluated in our study was $55.9 \pm 13.2$ years. Out of all patients, $55.1 \%(\mathrm{n}=387)$ were female and $44.9 \%(\mathrm{n}=315)$ were male, and $8.1 \%(\mathrm{n}=57)$ were diagnosed with type 1 diabetes and $91.9 \%(\mathrm{n}=645)$ with type 2 diabetes. The demographic and diabetes-related data of the patients are shown in Table 1 .

Musculoskeletal system problems were present in $45.9 \%(\mathrm{n}=322)$ of the patients included in the study. While 22.4\% $(\mathrm{n}=157)$ of the patients had a single finding, 23.5\% $(\mathrm{n}=155)$ had two and more findings together. The frequency of musculoskeletal system disorders were as follow: Carpal tunnel syndrome in $17.7 \%$ $(\mathrm{n}=124)$, Dupuytren's contracture in $14.4 \%(\mathrm{n}=101)$, limited joint mobility syndrome in $10.2 \%(\mathrm{n}=72)$, adhesive capsulitis in $13.8 \%(\mathrm{n}=97)$, flexor tenosynovitis in $2.8 \%(\mathrm{n}=20)$, diabetic foot in $6 \%(\mathrm{n}=39)$ and Charcot arthropathy in $1.7 \%(\mathrm{n}=12)$. The most common musculoskeletal disorder was carpal tunnel syndrome, and bilateral involvement of the disease was observed in $46.7 \%$ ( $\mathrm{n}=58$ ) of patients. Dupuytren's contracture was found to be more common in patients with type 2 diabetes compared to patients with type 1 diabetes ( $15 \%$ and $1.8 \%$, respectively), and this result was statistically significant $(\mathrm{p}=0.015)$. The relationship of musculoskeletal disorders with the type of diabetes is shown in Table 2 .

The relationship of the musculoskeletal disorders with age and the duration of diabetes was evaluated. There was a statistically significant relationship between Dupuytren's contracture $(p<0.001)$, LJMS $(p<0.001)$ and diabetic foot $(p=0.021)$ and the mean patient age. The duration of diabetes was associated with Dupuytren's contracture, adhesive capsulitis, carpal tunnel syndrome, flexor tenosynovitis, and limited joint mobility syndrome $(\mathrm{p}<0.005)$.

In our study, the relationship between HbA1c level and musculoskeletal disorders was demonstrated. HbA1c levels were higher in patients with musculoskeletal disorders, and there was a statistically significant difference in Dupuytren's contracture $(p<0.001)$, adhesive capsulitis $(p=0.048)$, and carpal tunnel syndrome $(p=$ 0.045). The relationship between musculoskeletal disorders and HbA1c is shown in Figure 1 .

The proteinuria level was also higher in patients with musculoskeletal manifestations. However, a statistically 
significant relationship with proteinuria was found only between flexor tenosynovitis and diabetic foot ( $\mathrm{p}$ $<0.05)$. An association between microvascular complications and musculoskeletal disorders was also found. A significant relationship between Dupuytren's contracture and diabetic foot with all of the microvascular complications was detected. A relationship between adhesive capsulitis and retinopathy was also found to be significant. Likewise, there was a substantial relationship of LJMS and flexor tenosynovitis with nephropathy. The relationship of musculoskeletal system disorders with microvascular complications, age, duration of diabetes, HbA1c, and proteinuria are shown in Table 3.

\section{Discussion}

Several musculoskeletal disorders have been associated with diabetes. The prevalence of musculoskeletal disorders in patients with diabetes has increased compared to the healthy population..$^{2,5-7}$ Musculoskeletal system disorders were detected in $322(45.9 \%)$ of the 702 diabetic patients evaluated in our study. The frequency of musculoskeletal system disorders ranged from $36 \%$ to $75 \%$ in other researches. ${ }^{2,8-10}$ The variability may be caused by the inclusion of different patient populations in those studies in terms of the distribution of diabetes duration and types.

Carpal tunnel syndrome (CTS) is a trap neuropathy that develops as a result of compression of the median nerve under the transverse carpal ligament at the wrist. Nerve compression can also stem from the thickening and abnormal growth of the connective tissue. In studies conducted in the literature, the frequency of carpal tunnel syndrome was found to vary between $5.3 \%$ and $41.7 \%^{2,7,9,11,12}$ In a study of 432 patients with diabetes by Kiani et al. ${ }^{12}$, the prevalence of CTS was found to be $8 \%$, and it was shown that there was a significant relationship between female gender and duration of diabetes. In the study of Khader et al. ${ }^{9}$, it was emphasized that obesity is related to CTS. In our research, the most common musculoskeletal finding was carpal tunnel syndrome $(17.7 \%)$, and there was a statistically significant correlation between HbA1c level and carpal tunnel syndrome $(p=0.045)$. In some studies, the relationship between CTS and microvascular complications has been investigated, and an association has been shown between CTS and retinopathy. ${ }^{9,13}$ In our study, there was no difference in the frequency of CTS with the presence of retinopathy and nephropathy, while it was observed that it was significantly more common in those with neuropathy (Table 3).

Dupuytren's contracture is a fibroproliferative disease of palmar and digital fascia, characterized by abnormal scar tissue formation in the palmar region of the hand. Its frequency was reported to be between $13 \%$ and $39 \%$ in patients with diabetes. ${ }^{5,14}$ In our study, the frequency of Dupuytren's contracture was found to be $14.4 \%$. Rajendran et al. ${ }^{7}$ reported a higher Dupuytren's contracture prevalence $(42 \%)$ in their study composed of 206 patients with type 2 diabetes. Similar to our results, the frequency of Dupuytren's contracture was found to be $13 \%$ in a study by Ramchurm et al. ${ }^{2}$ In the study of Pandey et al. ${ }^{13}$, Dupuytren's contracture was found to be 19\%, and the relationship with the HbA1c level was shown in patients. In our study, Dupuytren's contracture was found to be associated with the HbA1c level, as shown in Table 3. In studies conducted, Dupuytren's contracture was found to be related to the presence of neuropathy and retinopathy, which are microvascular complications. ${ }^{9,13}$ In our study, Dupuytren's contracture was found to be significantly associated with all microvascular complications.

Adhesive capsulitis is characterized by painful, gradual loss of active and passive shoulder motion resulting from fibrosis and contracture of the joint capsule. The prevalence of adhesive capsulitis differs in the research depending on the criteria used for diagnosis and the population investigated. This difference is thought to occur due to the fact that calcific tendinitis of the shoulder is not included in some studies since imaging technologies are not used. In a meta-analysis evaluating 18 studies by Zreik et al. ${ }^{15}$, the prevalence of adhesive capsulitis in diabetes was found to be five times higher than in the normal population. Its frequency varied between $19 \%$ and $29 \%$ in patients with diabetes. ${ }^{14,16}$ The frequency of adhesive capsulitis was found to be $13.8 \%$ in our study, and its prevalence was similar to the studies in the literature. Ardic et al. ${ }^{6}$ found the frequency of adhesive capsulitis as $12.8 \%$ in their research. In another study, the frequency of adhesive capsulitis was found to be $29 \%$, and the relationship with age and duration of diabetes was shown. At the same time, adhesive capsulitis was compared with other complications of diabetes and was associated with the presence of retinopathy. ${ }^{16,17}$ In our study, a significant relationship was found between adhesive 
capsulitis and neuropathy, and retinopathy (Table 3).

The limited joint mobility syndrome is characterized by flexion contractures, especially in small joints, and thickening and hardening of the skin on the back of the hand and fingers. Its prevalence varies between $8 \%$ and $60 \%$ in patients with diabetes. ${ }^{2,7-9,11,13}$ Mathew et al. ${ }^{10}$ found the frequency of LJMS as $8.06 \%$ in their study. Similarly, LJMS prevalence was found to be $10.2 \%$ in our study. In a study in which Frost et al. ${ }^{18}$ evaluated 335 patients with type 1 diabetes, the relationship between the presence of LJMS and nephropathy and retinopathy, especially in men, was demonstrated. When we evaluated our study in terms of microvascular complications, there was a significant relationship between LJMS and the presence of nephropathy

\section{(Table 3).}

Flexor tenosynovitis (Trigger Finger) is characterized by localized thickening, palpable nodule formation, and triggering during finger movements in the flexor tendon sheath. ${ }^{19}$ Yosipovitch et al. ${ }^{19}$ found flexor tenosynovitis to be $5 \%$. In our study, the frequency of flexor tenosynovitis was found to be $2.8 \%$. In the literature, there are studies reporting a higher rate of flexor tenosynovitis. ${ }^{2,6,11,19}$ Pandey et al. did not show a relationship between microvascular complications and flexor tenosynovitis, and Khader et al. associated retinopathy. ${ }^{9,13}$ In our study, there was no relationship between flexor tenosynovitis, neuropathy, and retinopathy, but a significant association was found with the presence of nephropathy (Table 3).

In patients with diabetes, Charcot arthropathy is a reduction in the normal afferent protective neural impulses, and therefore loss of protection from trauma to the joint leads to progressive, painless joint destruction. ${ }^{20}$ Charcot arthropathy is found to be in $0.1-5 \%$ of patients with diabetes. It is more common in type 2 diabetes and is equally common in men and women. The incidence increases with the duration of diabetes. ${ }^{21} \mathrm{In}$ our study, Charcot arthropathy was found to be $1.7 \%$. In a study published by Agrawal et al. ${ }^{8}$, the frequency of Charcot arthropathy was found to be $2.9 \%$. In the study conducted by Stuck et al. ${ }^{22}$, the data of 652 patients with diabetes diagnosed with Charcot arthropathy were evaluated, and it was emphasized that the duration of diabetes was associated with Charcot arthropathy. In the same study, the incidence of Charcot arthropathy increased in patients with neuropathy and those with HbA1c levels above 7\%. In our study, no relationship was found between metabolic parameters and microvascular complications in patients diagnosed with Charcot arthropathy.

The frequency of diabetic foot was found to be $6 \%$ in our study. In the study conducted by Ardic et al. ${ }^{6}$, the frequency of diabetes foot was reported to be $6.4 \%$ and was associated with retinopathy. In a study evaluating 811 patients with type 2 diabetes by Kumar et al. ${ }^{23}$ the prevalence of diabetic foot was found to be $5.3 \%$. In our study, the diabetic foot was associated with age and duration of diabetes. A significant correlation was shown between the mean fasting blood glucose and proteinuria levels of the metabolic parameters and the diabetic foot. The relationship between all microvascular complications and diabetic foot was observed

\section{(Table 3).}

Our study has some limitations. Firstly, we did not have a control group. Secondly, a relatively small number of patients with type 1 diabetes were included in our study. However, musculoskeletal disorders have been shown to occur more frequently in people with type 1 diabetes when compared with the non-diabetic subject. ${ }^{24}$ Next, we could not evaluate osteoarthritis and rheumatoid arthritis in this study.

On the other hand, the prospective nature of the study, evaluation of the patients by the same physician, and a relatively large number of the study population could be designated as the strengths of the current paper. Furthermore, our results have shed light on the pathogenesis of musculoskeletal disorders by means of assessing the relationship between metabolic parameters and microvascular complications.

Therefore, we suggest that further larger prospective studies with control groups are required to understand the mechanisms and prevention of these frequent but under-studied complications of type 1 and type 2 diabetes.

As a result of our study, $45.9 \%(\mathrm{n}=322)$ of the 702 diabetic patients showed signs of musculoskeletal 
system disorders. The most common disorders were carpal tunnel syndrome, Dupuytren's contracture, and adhesive capsulitis. Our study also draws attention to various risk factors that are effective in musculoskeletal system involvement in diabetes. These risk factors are age, duration of diabetes, HbA1c level as a marker of glycemic control, and presence of microvascular complications. A statistically significant relation was found between the duration of diabetes and all musculoskeletal disorders except Charcot arthropathy and between HbA1c level and Dupuytren's contracture, carpal tunnel syndrome, and adhesive capsulitis. In addition, the frequency of musculoskeletal disorders increased with the presence of microvascular complications.

\section{Acknowledgments}

Funding : This research did not receive any specific grant from funding agencies in the public, commercial, or not-for-profit sectors.

Conflicts of Interest/Competing Interests : The authors declare they have no conflict of interest

Availability of data and material : The authors confirm that the data supporting the findings of this study are available within the article and/or its supplementary materials.

Code availability : Not Applicable

Ethics approval: The study was approved by the Ethics Committee of Celal Bayar University, Faculty of Medicine.

Consent to participate: Informed consent was obtained from each of the participants

\section{References}

1. Diabetes Canada Clinical Practice Guidelines Expert Committee, Punthakee Z, Goldenberg R, Katz P Definition, Classification and Diagnosis of Diabetes, Prediabetes and Metabolic Syndrome. Can J Diabetes 2018; Apr; 42: Suppl (1): S10-S15. doi: 10.1016/j.jcjd.2017.10.003.

2. Ramchum N, Mashamba C, Leitch E Upper limb musculoskeletal abnormalities and poor metabolic control in diabetes. Eur J Intern Med 2009 ; 20: 718-21.

3. Crispin JC, Alcocer-Varela J Rheumatologic manifestations of diabetes mellitus. Am J Med 2003; 114: (9): 753-7.

4. Brownlee M, Cerami A, Vlassara H Advanced glycosylation end products in tissue and the biochemical basis of diabetic complications. N Engl J Med 1988; 318(20): 1315-21.

5. Lebiedz-Odrobina D, Kay J Rheumatic manifestations of diabetes mellitus. Rheum Dis Clin North Am 2010; 36(4): 681-99.

6. Ardic F, Soyupek F, Kahraman Y, Yorgancioglu R. The musculoskeletal complications seen in type 2 diabetics: predominance of hand involvement. Clin Rheumatol 2003; (22): 229-33.

7. Rajendran SR, Bhansali A, Walia R, Dutta P, Bansal V, Shanmugasundar G. Prevalence and pattern of hand soft-tissue changes in type 2 diabetes mellitus. Diabetes \& Metabolism 2011; 37(4): 312-7.

8. Agrawal RP, Gothwal S, Tantia P, et al. Prevalence of Rheumatological Manifestations in Diabetic Population from North-West India. Journal of the Association of Physicians of India 2014; 62(9): 788-92.

9. Mustafa KN, Khader YS, Bsoul AK, Ajlouni K. Musculoskeletal disorders of the hand in type 2 diabetes mellitus: prevalence and its associated factors. Int J Rheum Dis 2016; 19(7): 730-5.

10. Mathew AJ, Nair JB, Pillai SS Rheumatic-musculoskeletal manifestations in type 2 diabetes mellitus patients in south India. Int $\mathrm{J}$ Rheum Dis 2011;14: 55-60.

11. Al-Matubsi HY, Hamdan F, Alhanbali OA, Oriquat GA, Salim M. Diabetic hand syndromes as a clinical and diagnostic tool for diabetes mellitus patients. Diabetes Res Clin Pract 2011; 94(2): 225-9. 
12.Kiani J, Gohannifar H, Moghimbeigi A, Azizkhani H. Prevalence and risk factors of five most common upper extremity disorders in diabetics. J Res Health Sci 2014; 14(1): 92-5.

13.Pandey A, Usman K, Reddy H, Gutch M, Jain N, Qidwai Sa. Prevalence of hand disorders in type 2 diabetes mellitus and its correlation with microvascular complications. Ann Med Health Sci Res 2013; 3(3): $349-54$.

14. Bañón S, Isenberg DA. Rheumatological manifestations occurring in patients with diabetes mellitus. Scand J Rheumatol 2013; 42(1): 1-10.

15. Zreik NH, Malik RA. Charalambous CP Adhesive capsulitis of the shoulder and diabetes: a meta-analysis of prevalence. Muscles, Ligaments and Tendons J 2016; 6(1): 26-34.

16. Balci N, Balci MK, Tüzüner S. Shoulder adhesive capsulitis and shoulder range of motion in type II diabetes mellitus: association with diabetic complications. J Diabetes Complications 1999; 13(3): 135-40.

17. Arkkila ETP, Kantola MI, Viikari SAJ, Ronnemaa T. Shoulder capsulitis in type I and II diabetic patients: association with diabetic complications and related diseases. Ann Rheum Dis 1996; 55: 907-14.

18. Frost D, Beischer W. Limited joint mobility in type 1 diabetic patients: associations with microangiopathy and subclinical macroangiopathy are different in men and women. Diabetes Care 2001; 24: 95-9.

19. Yosipovitch G, Yosipovitch Z, Karp M, Mukamel M. Trigger finger in young patients with insulin dependent diabetes. J Rheumatol 1990; 17(7):951-2.

20. Rogers LC, Frykberg RG, Armstrong DG, Boulton AJM, Edmonds M, Ha Van G. The Charcot foot in diabetes. Diabetes Care 2011; 34(9): 2123-9.

21.Hartemann-Heurtier A, Van Ha G, Grimaldi A. The Charcot foot. Lancet 2002 ; (360): 1776-9.

22.Stuck RM, Sohn MW, Budiman-Mak E, Lee T, Weiss KB. Charcot arthropathy risk elevation in the obese diabetic population. Am J Med 2008; 121(11): 1008-14.

23.Kumar S, Ashe HA, Parnell LN, Fernando DJ, Tsigos C, Young RJ. The prevalence of foot ulceration and its correlates in type 2 diabetic patients: a population-based study. Diabet Med 1994; 11(5): 480-4.

24. Larkin ME, Barnie A, Braffett BH, Cleary PA, Diminick L, Harth J. Diabetes Control and Complications Trial/Epidemiology of Diabetes Interventions and Complications Research Group. Musculoskeletal complications in type 1 diabetes. Diabetes Care 2014; 37:1863-9.

Table 1. Demographic and diabetes-related data of the patients

Gender

Age

Duration of Diabetes (years)

Body Mass Index $\left(\mathrm{kg} / \mathrm{m}^{2}\right)$

Type of Diabetes

Concomitant disease
Female

Male

[?] 50

$>50$

[?] 5

5-10

$>10$

[?]25

$>25$

Type 1

Type 2

Hypertension

No hypertension 


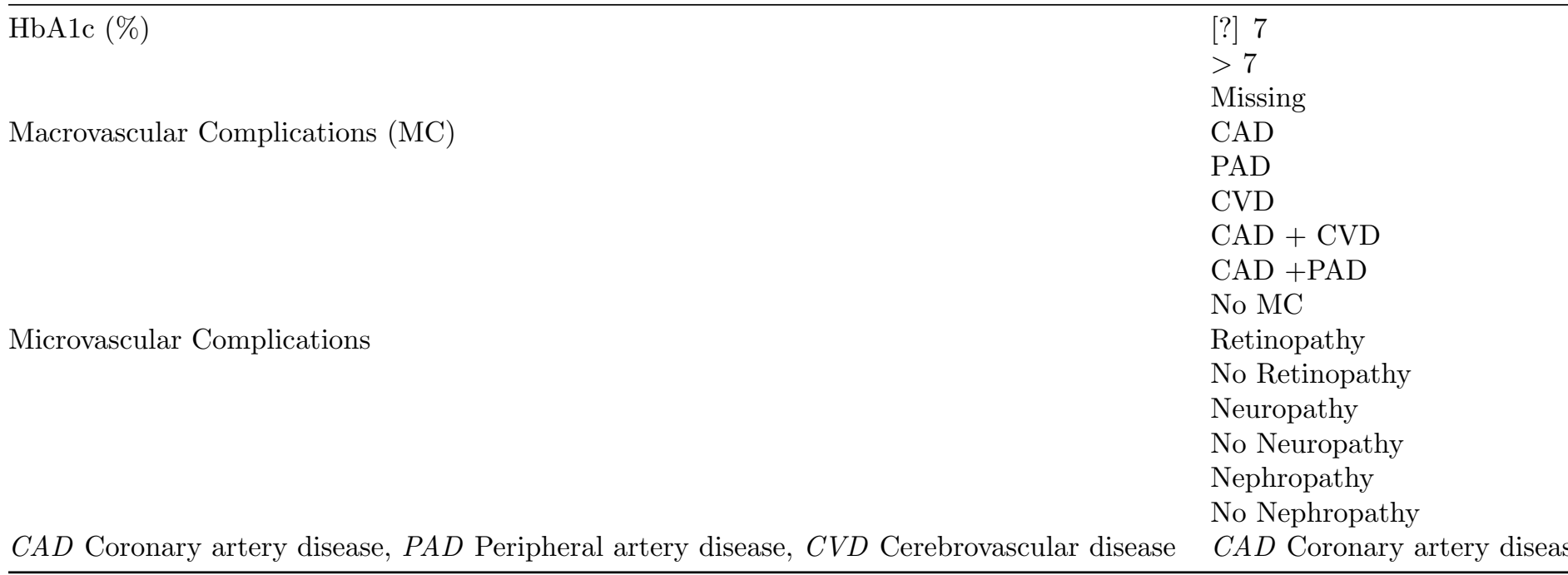

Table 2. The relationship of musculoskeletal disorders with the type of diabetes

\begin{tabular}{|c|c|c|c|c|c|}
\hline Musculoskeletal disorders & Type of Diabetes & Type of Diabetes & Type of Diabetes & Type of Diabetes & Total $(n=702)$ \\
\hline & Type $1(n=57)$ & Type $1(\mathrm{n}=57)$ & Type $2(\mathrm{n}=645)$ & Type $2(\mathrm{n}=645)$ & \\
\hline & $\mathbf{n}$ & $\%$ & $\mathbf{n}$ & $\%$ & $\mathbf{n}$ \\
\hline Dupuytren's contracture & 1 & 1,8 & 100 & 15 & 101 \\
\hline Adhesive capsulitis & 2 & 3 & 95 & 14 & 97 \\
\hline Carpal tunnel syndrome & 4 & 7,1 & 120 & 18,6 & 124 \\
\hline Flexor tenosynovitis & 1 & 1,8 & 19 & 2,9 & 20 \\
\hline LJMS & 3 & 5 & 71 & 11 & 74 \\
\hline Charcot arthropathy & 1 & 1,8 & 11 & 1,7 & 12 \\
\hline Diabetic foot & 3 & 5 & 39 & 6 & 42 \\
\hline Gout & 2 & 3.5 & 9 & 1.3 & 11 \\
\hline
\end{tabular}

*Pearson chi-square

Table 3. The relationship of musculoskeletal system disorders with microvascular complications, age, duration of diabetes, $\mathrm{HbA1c}$, and proteinuria

\begin{tabular}{|c|c|c|c|c|c|c|c|c|c|c|}
\hline $\begin{array}{l}\text { Musculosk } \\
\text { Disorders }\end{array}$ & $\begin{array}{l}\text { endtadculoske } \\
\text { Disorders }\end{array}$ & $\begin{array}{l}\text { elegal } \\
\text { (years) }\end{array}$ & $\begin{array}{l}\text { Duration } \\
\text { of Dia- } \\
\text { betes } \\
\text { (years) }\end{array}$ & $\begin{array}{l}\text { Duration } \\
\text { of Dia- } \\
\text { betes } \\
\text { (years) }\end{array}$ & $\begin{array}{l}\text { HbA1c } \\
(\%)\end{array}$ & $\begin{array}{l}\mathrm{HbA1c} \\
(\%)\end{array}$ & $\begin{array}{l}\text { Proteinuria } \\
\text { (mg/day) }\end{array}$ & $\begin{array}{l}\text { Retino } \\
\text { apathy } \\
(\mathrm{n})\end{array}$ & $\begin{array}{l}\text { Neuro } \\
\text { pathy } \\
(\mathrm{n})\end{array}$ & $\begin{array}{l}\text { Neuro } \\
\text { pathy } \\
(\mathrm{n})\end{array}$ \\
\hline \multirow[t]{4}{*}{$\begin{array}{l}\text { Dupuytren } \\
\text { contractur }\end{array}$} & $\begin{array}{l}\text { 'sio } \\
\mathrm{e}^{*}\end{array}$ & 55 & 55 & 8,6 & 8 & 92,3 & 92,3 & 143 & 272 & 152 \\
\hline & Unilateral & 61,5 & 61,5 & 11,4 & 9 & 114,7 & 114,7 & 24 & 34 & 16 \\
\hline & Bilateral & 65,3 & 65,3 & 13,8 & 9,1 & 136 & 136 & 13 & 20 & 13 \\
\hline & $P$ & $<0.001$ & $<0.001$ & $<0.001$ & $<0.001$ & 0,829 & 0,829 & $<0.001$ & $<0.001$ & 0,040 \\
\hline $\begin{array}{l}\text { Carpal } \\
\text { tunnel } \\
\text { syndrome* }\end{array}$ & No & 55,6 & 55,6 & 8,6 & 8,0 & 98,6 & 98,6 & 148 & 256 & 156 \\
\hline
\end{tabular}




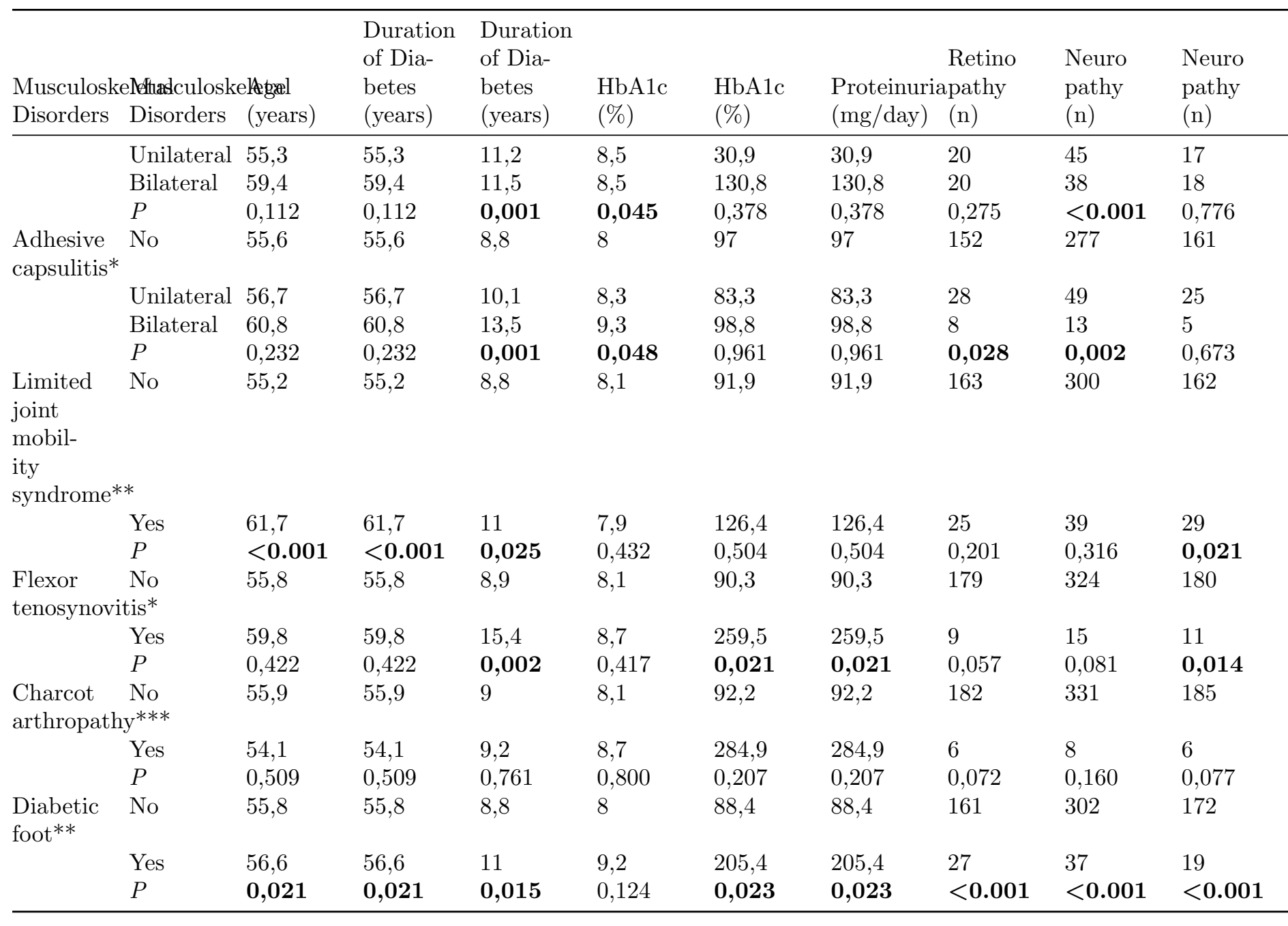

* Oneway ANOVA

** Student's t-test

***Mann-Whitney U Test

( $P$-value of less than 0.05 was accepted as statistically significant)

Figure Legend

Fig. 1. The relationship between musculoskeletal disorders and HbA1c

Hosted file

Figure 1.docx available at https://authorea.com/users/431050/articles/534579-themusculoskeletal-disorders-in-diabetic-patients-and-the-evaluation-of-their-relationshipwith-metabolic-parameters-and-microvascular-complications

Hosted file

Table 1.docx available at https://authorea.com/users/431050/articles/534579-themusculoskeletal-disorders-in-diabetic-patients-and-the-evaluation-of-their-relationshipwith-metabolic-parameters-and-microvascular-complications 


\section{Hosted file}

Table 2.docx available at https://authorea.com/users/431050/articles/534579-themusculoskeletal-disorders-in-diabetic-patients-and-the-evaluation-of-their-relationshipwith-metabolic-parameters-and-microvascular-complications

\section{Hosted file}

Table 3.docx available at https://authorea.com/users/431050/articles/534579-themusculoskeletal-disorders-in-diabetic-patients-and-the-evaluation-of-their-relationshipwith-metabolic-parameters-and-microvascular-complications 